\title{
Ueber die Methylnaphtaline;
}

von

\section{Gustav Wendt. ${ }^{1}$ )}

Als Ausgangsmaterial dienten ca. 4 Kilo eines Gemenges der beiden Isomeren, geliefert von der J. Rütgers'schen Fabrik in Wien-Angern. Dieses Rohprodukt, aus dem Kreosotöl des Handels durch vielfache Destillation und unter Anwendung der üblichen Reinigungsmethoden gewonnen, destillirte zum allergrössten Theile zwischen $239^{\circ}-243^{\circ}$ und zeigte damit den von K. E. Schulze in Mannheim ${ }^{2}$ ) für das Gemenge der Isomeren gefundenen Siedepunkt.

Das dünnflüssige, bräurliche Oel wird allmählich dunkler bis dunkelrothbraun. Bei der Herstellung von Sulfosäuren gelang es, ein einfaches Mittel zu finden, um die lichtempfindliche Beimengung zu entfernen. Schüttelt man nämlich das Oel kurze Zeit mit dem gleichen Volumen kalter Schwefelsäure (1 Volumen Schwefelsäure von $66^{\circ}$ Baumé +1 Volumen Wasser), so färbt sich das Oel und die Schwefelsäure dunkelbraun.

Sättigt man die gefärbte Schwefelsäure mit Baryumcarbonat, so resultirt ein in Wasser lösliches, in breiten, flachen Nadeln krystallisirendes organisches $\mathrm{Salz}$, welches 52,87\% Baryum enthält. (Das Salz der Monosulfonsäure des Methylnaphtalins z. B. enthält 23,66\% Baryum.) Das Salz machte sich bei der Herstellung der Sulfosäuren durch seine gute

1) cf. Gustav Wendt: Von den beiden isomeren Methylnaphtalinen. Inaug.-Dissert., Berlin 1891. Siehe ferner H. Wichelhaus: Ueber die Methylnaphtaline, Ber. 24, 3918. In der letztgenannten Arbeit wird keine Notiz von der Dissertation genommen, obwohl dieselbe nicht nur vorher - und zwar im Institute und unter der Oberleitung des Herrn Prof. Wichelhaus - ausgeführt, sondern auch ca. 4 Monate früher veroffentlicht wurde. Deshalb erscheint es wohl angezeigt, insbesondere die Angaben in den Berichten zu ergänzen, umso mehr als z. B. das vom Verfasser der Dissertation ohne jede Beihülfe und nach eigener Methode gereinigte und getrennte Rohmaterial von Herrn Prof. Wichelhaus zwar erwähnt und offenbar benutzt worden ist, jedoch ohne Namennennung des Bearbeiters.

$\left.{ }^{2}\right)$ Ber. 17, (1884) 842 u. 1528. 
Krystallisation bemerkbar im Gegensatze zu den Salzen der Sulfonsäuren, die durchweg schlecht krystallisiren, sowie ferner dadurch, dass die ersten Analysen der Barytsalze stets etwas zu viel Baryum aufwiesen. Der Baryumgehalt von 52,87\% ermuthigte nicht zu näherer Untersuchung, zumal der Körper nur in sehr geringer Menge vorhanden war.

Wird nun das Oel einige Male mit der Schwefelsäure ausgeschüttelt und schliesslich mit Wasserdampf übergetrieben, so erhält man es fast farblos und unveränderlich an Luft und Licht.

Durch längere Berührurıg mit wasserfreiem Chlorcalcium. wird es gut getrocknet und verliert dabei die blüuliche Fluorescenz, die ihm sonst stets anhaftet. Wenn Hr. Wichelhaus sein Methylnaphtalin „fluorescirend“ nennt, so wird dadurch angedeutet, dass das betreffende Oel sehr feucht oder sehr unrein war.

Noch heute sehen ca. 100 Grm. des gereinigten und getrockneten Oeles (und zwar $\alpha$-Methylnaphtalin) genau so aus wie vor ca. 3 Jahren: Hellgelb, krystallklar, in Folge der starken Lichtbrechung mit farbigen Reflexen versehen, ohne jede Spur von Fluorescenz.

Die Hauptaufgabe war die Trennung der Isomeren, die bisher wegen der Gleichheit der Siedepunkte und der Aehnlichkeit aller Eigenschaften nicht durchgeführt werden konnte. Nur das feste $\beta$-Methylnaphtalin wurde bisher rein gewonnen, während vom flüssigen $c$-Methylnaphtalin selbst K.E. Schulz $\mathrm{e}^{1}$ ), der das Meiste für die Kenntniss der Methylnaphtaline gethan hat, nur angeben kann, dass sein flüssiges Methylnaphtalin bei $-15^{0}$ "möglichst" von allen krystallinischen Substanzen befreit wurde.

Es galt also, eine allgemeine Methode zu finden, um ein Oel vollständig von gelösten krystallinischen Substanzen frei zu machen, während man bisher nur im Stande war, gelöste krystallinische Substanz aus Oel rein zu gewinnen.

Mein Apparat zur Trennung von Kohlenwasserstoffen durch fractionirtes Schmelzen ist in der Dissertation abgebildet

1) Ber. 17, 844 . 
und genau beschrieben. ${ }^{1}$ ) Das Princip desselben ist Folgendes: Das zu trennende Gemenge wird soweit abgeküblt, dass es durchweg hart und fest ist und $z$ war in einem vor äusserer Temperatureinwirkung gut geschützten Behälter. Ueberlässt man darauf das Gemenge sammt den umgebenden Abkühlungsflüssigkeiten sich selbst, so wird die Temperatur im Behälter, vorausgesetzt, dass die umgebende Temperatur höher ist, allmählich steigen. Sobald nun der niedrigste Schmelzpunkt eines Körpers in dem Gemenge erreicht ist, wird derselbe zu schmelzen beginnen und zwar allmählich, da die nöthige Schmelzwärme nur langsam von aussen hinzugeführt wird. Während dieses Schmelzens ist der entstehenden Flüssigkeit Gelegenheit gegeben, durch ein am Boden befindliches Sieb mit Leinwandfilter abzutropfen. Bei der ungefähren Schmelztemperatur wird der schmelzende Körper eine ganz geringe oder gar keine Lösungsfähigkeit für beigemengte feste Substanzen, resp. Lösungswärme besitzen. Durch mehrfaches Wiederholen des dargestellten Processes tropft zuletzt ein von den höher schmelzenden Substanzen völlig freies Oel ab bei constantem Schmelzpunkt. Durch jedwede Saug- oder Druckvorrichtung wird die Lösungsfähigkeit, besonders dicht am Filter, resp. beim Durchgange durch die Poren des Filters vollständig verändert, sodass eine solche Vorrichtung ganz ausgeschlossen werden muss. Falls nicht zu viel feste Substanz vorhanden ist, gebt das Abtropfen in einem zweckmässigen Apparate auch schnell von statten.

Von ca. 2140 Grm. des mehrfach destillirten und, wie vorher angegeben, gereinigten Ausgangsmaterials wurden durch wiederholtes fractionirtes Schmelzen erhalten: $1483 \mathrm{Grm}$. Oel, $555 \mathrm{Grm}$. festes Produkt und ca. $90 \mathrm{Grm}$. Oel vom letzten Abpressen des festen Produktes bei $-4^{0}$. Diese letzten ca. $90 \mathrm{Grm}$. können, wie durch weiteres Ausfrieren festgestellt wurde, als ungefähr aus gleichen Theilen festen und flüssigen Methylnaphtalins bestehend angesehen werden. Sie lohnten die Trennung nicht mehr, da dieselbe zweckmässig nur mit grösseren Mengen vorzunehmen ist, und wurden $\mathrm{zu}$ andern Portionen behufs fractionirten Schmelzens zugegeben.

1) Inaug.-Dissert. S. $20 \mathrm{ff}$, 
Die 1483 Grm. Oel waren so gut wie frei von festen Produkten. Eine mittlere Portion wurde für sich aufgefangen. Das Ganze wurde getrocknet und zeigte durchweg constante Schmelz- und Siedepunkte.

In den $555 \mathrm{Grm}$. festen Produktes waren noch wägbare Mengen Oel enthalten, was sich z. B. beim Stehen in der Wärme zeigte. Sie wurden durch mehrfaches Umkrystallisiren aus Alkohol gereinigt.

Das Verhältniss des festen zum flüssigen Theile ergiebt sich hiernach als ca. 2 zu 5 . In Gegensatze hierzu giebt K. E. Schulze ${ }^{1}$ ) dieses Verhältniss wie ca. 3 zu 2 an. $\mathrm{Er}$ sagt, und diese Angabe ist die einzige über den Procentgehalt im Steinkohlentheer: „Im Kreosotöl des Handels, welches bei über $200^{\circ}$ destillirt, sind durchschnittlich $6 \%$ enthalten. Durch Extraction der Carbolsäure und Rectificiren des Oeles, wobei die Fraction von $200^{\circ}-300^{\circ}$ aufgefangen wird, gelangt man $\mathrm{zu}$ einem Produkt, welches $10 \%$ der in Rede stehenden Körper enthält und zwar etwa $4 \% \quad c$-(fiüssiges) und $6 \% \beta$-(festes) Methylnaphtalin."

Diese grosse Verschiedenheit zwischen dem hier mitgetheilten Befunde und $\mathbf{S c h u l z e}$ 's Angabe lässt sich nicht dadurch allein erklären, dass Sehulze's flüssiges Methylnaphtalin unzweifelhaft feste Produkte enthielt, noch ferner dadurch, dass Schulze's Kreosotöl ein anderes war, wie das der Fabrik in Wien-Angern, sondern allein dadurch, dass Schulze die ganze in seinem Kreosotöl, enthaltene Menge des Methylnaphtalins, also $10 \%$ des Kreosotöls prüfte, während das von Wien-Angern gelieferte Material nur einen Theil darstellte.

$\alpha$-Methylnaphtalin ist ein schwach gelbliches, leichtflüssiges Oel und unveränderlich durch Luft und Licht. Der Schmelzpunkt liegt bei $-22^{\circ}$, der Siedepunkt zwischen $240^{\circ}-243^{\circ}$ (Quecksilbersäule im Dampf, Barometerstand $759 \mathrm{Mm}$.).

Das spec. Gew. wurde mit der Mohr'schen Waage = 1,0005 bei $19^{\circ}$ gefunden. $^{2}$ ) Die Elementaranalyse ergab:

1) Ber. 17, 1528.

2) Schulze fand gleichfalls den Siedepunkt bei $240^{\circ}-243^{\circ}$. (Ber. 17, 843.) Vom spec. Gew. giebt er leider nur das des Gemenges der Isomeren $=1,006$ bei $15^{\circ}$. Dagegen stellten Fittig und Remsen bei 
0,1560 Grm. Substanz lieferten 0,5312 Grm. Kohlensäure und 0,1016 Grm. Wasser.

$\begin{array}{ccc} & \text { Berechnet für } \mathrm{C}_{11} \mathrm{H}_{10}: & \text { Gefunden: } \\ \mathrm{C} & 92,96 & 92,80 \% \\ \mathrm{H} & 7,04 & 7,23 \% .\end{array}$

Das Oel löst sich leicht in Alkohol und Aether, sehr wenig in Wasser und ist mit Wasserdampf flüchtig. Es hat selbst ein starkes Lösungsvermögen. Es brennt mit leuchtender Flamme, leitet die Wärme schlecht, bricht das Licht sehr stark und zeigt keine Fluorescenz. Es besitzt einen eigenthümlichen aromatischen und an Naphtalin erinnernden Geruch, sowie einen etwas brennenden, seifigen Geschmack.

$\beta$-Methylnaphtalin krystallisirt aus Alkohol in durchsichtigen Blättern, ist leicht löslich in Lösungsmitteln wie Alkohol, Aether, Chloroform, Benzol etc., schmilat bei $32,5^{\circ}$ und siedet zwischen $241^{\circ}-242^{\circ}$. (Quecksilbersäule im Dampf, Barometerstand $759 \mathrm{Mm}^{1}{ }^{1}$ ) Die Elementaranalyse ergab:

0,1330 Grm. Substanz lieferten 0,4526 Grm. Kohlensäure und 0,0827 Grm. Wasser.

$\begin{array}{ccc} & \text { Berechnet für } \mathrm{C}_{11} \mathrm{H}_{10}: & \text { Gefunden: } \\ \mathrm{C} & 92,96 & 92,80 \% \\ \mathrm{H} & 7,04 & \mathbf{7 , 1 5} \%\end{array}$

Die sonstigen Eigenschaften sind so ziemlich dieselben, wie beim flüssigen Methylnaphtalin, nur dass das feste in viel geringerem Grade mit Wasserdämpfen flüchtig ist. $\mathrm{Zu}$ bemerken wäre noch, dass der Körper nicht zur Sublimation zu bringen war, obwohl er doch, wie der starke Geruch verräth, bereits bei gewöhnlicher Temperatur verdunstet. Durch ganz

der ersten Auffindung des Kohlenwasserstoffes durch Synthese (Ann. Chem. 1a5, 114) das spec. Gew. ihres flüssigen Methylnaphtalins $=1,0287$ bei $11,5^{\circ}$ fest und ferner den Siedepunkt bei $231^{\circ}-232^{\circ}$. Schulze wiederholte die Fittig'sche Synthese und fand wiederum $240^{\circ}-243^{\circ}$.

1) Schulze (ber. 17, 843) und Léon Roux (bei der Synthese Ann. Chim. [6] 12, 289) fanden gleichfalls den Schmelzpunkt bei $32,5^{\circ}$. Schulze bestimmte auch den Siedepunkt bei $241^{\circ}-242^{\circ}$. Dagegen fanden Fittig und Liebmann (Ber, 20, 3182) den Schmelzpunkt bei $37^{\circ}-38^{\circ}$. Ihr $\beta$-Methylnaphtalin war aus Methylnaphtolen gewonnen durch Destillation über erhitztem Zinkstaub. Die Methylnaphtole, deren Eigenschaften noch nicht näher festgestelit worden sind, stammten aus den Destillationsprodukten von Phenylhomoparaconsäure und Phenylisohomoparaconsäure.

Journal f. pralt. Chemie [2] Bd. 46. 
gelindes Erwärmen auf lauwarmem Wasser wird dies Verdunsten sehr verstärkt.

Dass die Methylnaphtaline sehr reactionsfähige Körpersind, zeigt sich schon beim Sulfoniren. A us vielen Versuchen - es wurden z. B. allein von $\alpha$-Methylnaphtalinsulfonsäuren 15 Barytsalze auf ihren Barytgehalt geprüft - ergab sich Folgendes: Schon bei gewöhnlicher Temperatur gehen die einfach methylirten Naphtaline (möglichst trocken, da der Wassergehalt die Ausbeute sehr verringert) durch lange $\mathrm{Be}-$ rührung, besser aber durch Schütteln mit genau 1 Mol. concentrirter Schwefelsäure zum grössten Theile in Monosulfonsäuren über. Bei vielen andern wie den gegebenen Volumen- und Temperaturverhältnissen wurden stets Mischungen von Mono-, Di- und Trisulfonsäuren erhalten. Schon durch Schütteln mit $2 \mathrm{Mol}$ conc. Schwefelsäure bei gewöhnlicher Temperatur trat höhere Sulfonirung ein. Bei jedem Versuch konnten mit Sicherheit stets zwei Sulfonsäuren unterschieden werden, zunächst durch die verschiedene Löslichkeit ihrer Baryt- und Bleisalze. In der Kälte, resp. bei gewöhnlicher Temperatur und etwas Küblung während des Schüttelns entstanden fast ausschliesslich die leichter löslichen Säuren, resp. Salze und nur Spuren der weniger löslichen Salze. Der Länge und Höhe des Erwärmens entsprach die Quantität der schwerer löslichen Salze. Die freien Säuren unterscheiden sich besonders dadurch von einander, dass die des leichter löslichen Salzes an der Luft zerfliesslich, die des schwerer löslichen Salzes an der Luft nicht zerfliesslich sind.

Aus den Baryt- und Bleisalzen wurden stets drei Theile gewonnen, um das Salz, welches sich zuerst absetzte, von dem in der letzten Mutterlauge enthaltenen völlig zu trennen. Was beim Abdampfen der Mutterlauge zuerst ausfiel, wurde für sich auf dem Filter gesammelt und danach ein- bis zweimal mit einer zur Lösung ungenügenden Menge Wasser stehen gelassen. So konnte jede Spur dér leichter löslichen Salze entfernt werden. Zweitens wurde die Hauptmasse zur Krystallisation gebracht. Endlich diente die letzte Mutterlauge, welche event, zuvor entfärbt wurde, zur Darstellung des letzten, leicht löslichen Salzes.

Die Löslichkeit der beiden isomeren Barytsalze der Mono- 
sulfonsäuren verhält sich wie ca. 1 zu 6 sowohl beim $\iota$ - wie beim $\beta$-Methylnaphtalin. Die Löslichkeit der Barytsalze des $\beta$-Methylnaphtalins ist ungefähr nur halb so gross wie beim c-Methy Inaphtalin.

In Betreff der Krystallisation der freien Säuren, sowie der Baryt-, Blei-, Kali- und Natronsalze ist zu bemerken, dass diese Körper geringe Krystallisationsfähigkeit besitzen, dass sie jedoch alle, besonders beim Arbeiten mit grösseren Mengen und unter dem Mikroskope eine bestimmte, von einander verschiedene Structur zeigen, die meist aus kleinen Kügelchen besteht, resp. warzenförmig gestaltet ist.

$\alpha$-Methylnaphtalinmonosulfonsaurer Baryt I ist das letzte, (siehe oben) leicht lösliche Salz. Sämmtliche Barytsalze verloren ihr Krystallwasser erst durch andauerndes Trocknen bei $140^{\circ}-150^{\circ}$.

Die Analyse des trocknen Salzes ergab:

$0,3760 \mathrm{Grm}$. Substanz lieferten 0,15056 Grm. Baryumsulfat.

Berechnet für $\mathrm{C}_{22} \mathrm{H}_{18} \mathrm{~S}_{2} \mathrm{O}_{6} \mathrm{Ba}$ :

$\mathrm{Ba}$ 23,66

Gefunden: $23,54 \%$.

Die Krystallwasserbestimmung ergab:

0,1398 Grm. Substanz verloren 0,0134 Grm. Wasser, entsprechend $9,6 \%$. 3 Mol. Krystallwasser verlangen 9,3\% Wasser. Die Formel des krystallisirten Salzes ist demnach:

$$
\left(\mathrm{C}_{10} \mathrm{H}_{6} \mathrm{CH}_{3} \mathrm{SO}_{3}\right)_{2} \mathrm{Ba}+3 \mathrm{H}_{2} \mathrm{O} \text {. }
$$

Zur Löslichkeitsbestimmung wurde die Menge des (über Schwefelsäure getrockneten) Salzes gewogen, welche in $10 \mathrm{Ccm}$. der bei $22^{\circ} \mathrm{ge}-$ sättigten Lösung enthalten war. Es wurden erhalten $0,2072 \mathrm{Grm}$; d. h. ein Theil wird von ca. 50 Theilen Wasser von $22^{\circ}$ gelöst.

Die Blei-, Kali- und Natronsalze krystallisiren ähnlich wie das Baryumsalz und sind im Wasser gut löslich.

Ein Monosulfonchlorid der letzten Säure des $\iota$-Methylnaphtalins (durch Erwärmen mit $\mathrm{PCl}_{3}$ erhalten) sowie ferner: das Monosulfonamid - durch Umsetzen des Chlorids mit Ammoniumcarbonat - konnte nicht in einem zur Analyse geeignetem Zustande erhalten werden. Beide Körper waren bräunliche, dickflüssige Oele, aus denen sich nach einiger Zeit unter dem Exsiccator in der Kälte einige wenige Krystalle ausschieden, die sich jedoch auch nach Monaten nicht vermehrt hatten.

a-Methylnaphtalinmonosulfonsaurer Baryt II ist das erwähnte erste, schwer lösliche Salz. 
Die Analyse ergab:

0,3548 Grm. Substanz lieferten 0,14266 Grm. Baryumsulfat.

Berechnet für $\mathrm{C}_{22} \mathrm{H}_{18} \mathrm{~S}_{2} \mathrm{O}_{6} \mathrm{Ba}$ : Gefunden:

$\mathrm{Ba} 23,66 \quad 23,61 \%$.

Die Krystallwasserbestimmung ergab:

0,3504 Grm. Substanz verloren 0,0318 Grm. Wasser, entsprechend $9 \%$ Wasser. 3 Mol. Krystallwasser verlangen 9,3\%.

Die Formel des krystallisirten Salzes ist demnach:

$$
\left(\mathrm{C}_{20} \mathrm{H}_{6} \mathrm{CH}_{3} \mathrm{SO}_{3}\right)_{2} \mathrm{Ba}+3 \mathrm{H}_{2} \mathrm{O} \text {. }
$$

Es krystallisirt in runden Kügelchen, die oft zu geraden Reihen geordnet sind.

Zur Löslichkeitsbestimmung wurde die Menge des (über Schwefelsäure getrockneten) Salzes gewogen, welche in $10 \mathrm{Ccm}$. der bei $22^{\circ} \mathrm{ge}$ sättigten Lösungen enthalten war. Es wurden erhalten:

0,0326 Grm., d. h. 1 Thl. wird von ca. 310 Thln. Wasser von $22^{\circ}$ gelöst.

$\beta$-Methylnaphtalinmonosulfonsaurer Baryt I. Es ist das letzte, (siehe früher) leicht lösliche Salz.

Die Analyse ergab:

$0,3247 \mathrm{Grm}$. Substanz lieferten 0,13056 Grm. Baryumsulfat.

Berechnet für $\mathrm{C}_{22} \mathrm{H}_{18} \mathrm{~S}_{2} \mathrm{O}_{6} \mathrm{Ba}: \quad$ Gefunden:

$\mathrm{Ba} 23,66 \quad 23,62 \%$.

Die Krystallwasserbestimmung ergab:

$0,1234 \mathrm{Grm}$. Substanz verloren 0,0134 Grm. Wasser, entsprechend $10 \%$ Wasser. 3 Mol. Krystallwasser verlangen 9,3\% Wasser. Die Formel des krystallisirten Salzes ist demnach $\left(\mathrm{C}_{10} \mathrm{H}_{6} \mathrm{CH}_{3} \mathrm{SO}_{3}\right)_{2} \mathrm{Ba}+3 \mathrm{H}_{2} \mathrm{O}$.

Es krystallisirt in Klümpchen, die bei guter Ausbildung sich unter dem Mikroskop als aus kleinen Nadeln bestehend zeigen.

Zur Löslichkeitsbestimmung wurde die Menge des (übcr Schwefelsäure getrockneten) Salzes gewogen, welche in $10 \mathrm{Ccm}$. der bei $22^{\circ} \mathrm{ge}$ sättigten Lösung enthalten war.

Es wurde erhalten:

0,1120 Grm., d. h. 1 Thl. wird von ca. 90 Thln. Wasser von $22^{\circ}$ gelöst.

Die Blei-, Kali- und Natronsalze krystallisiren ähnlich wie das Baryumsalz und sind in Wasser löslich.

$\beta$-Methylnaphtalinmonosulfonsaurer Baryt II ist das erste, schwer lösliche Salz.

Die Analyse ergab:

0,2862 Grm. Substanz lieferten 0,11436 Grm. Baryumsulfat.

Berechnet für $\mathrm{C}_{22} \mathrm{H}_{18} \mathrm{~S}_{2} \mathrm{O}_{6} \mathrm{Ba}$ :

$\mathrm{Ba}$

23,66

Gefunden:

$23,48 \%$.

Die Krystallwasserbestimmung ergab: 
0,1734 Grm. Substanz verloren 0,0053 Grm. Wasser, entsprechend $3 \%$ Wasser. ${ }^{1}$ ) 1 Mol. Krystallwasser verlangt 3,3\%.

Die Formel des krystallisirten Salzes ist demnach:

$$
\left(\mathrm{C}_{10} \mathrm{H}_{6} \cdot \mathrm{CH}_{3} \cdot \mathrm{SO}_{3}\right)_{2} \mathrm{Ba}+\mathrm{H}_{2} \mathrm{O} \text {. }
$$

Es zeigt sich unter dem Mikroskop als Haufwerk von Nadeln.

Zur Löslichkeitsbestimmung wurde die Menge des (über Schwefelsäure getrockneten) Salzes gewogen, welche in $10 \mathrm{Ccm}$. der bei $22^{\circ}$ gesättigten Lösungen enthalten war. Es wurden erhalten:

0,0190 Grm., d. h. 1 Thl, wird von rund 530 Thln. Wasser von $22^{0}$ gelöst.

Zum Schlusse sei bemerkt, dass der Theil der Arbeit, welcher die längste Zeit in Anspruch genommen hat, dass die Naphtolschmelze weder bei den bisher üblichen noch bei vielfach geänderten Volumen-, Temperatur- und Druckverhältnissen Produkte lieferte, aus denen reine, farblose Methylnaphtole gewonnen werden konnten.

Wenn auch bei gewissen Verhältnissen die Ausbeuten quantitativ gute waren, gelang es doch auf keine Weise, trotz vielfacher Mühe, aus den dunkeln, resp. schwarzen, theilweise zähen Massen farblose, krystallinische Methylnaphtole zu erhalten. Dass aber neue Naphtole vorlagen, konnte besonders durch charakteristische Azofarbstoff-Reactionen erkannt werden.

Herr Prof. Wichelhaus setzt sich in der zu Anfang erwähnten Abhandlung mehrfach nicht nur mit meiner Dissertation, sondern mit allen andern Autoren in betreff der Methylnaphtaline in Widerspruch. $\mathrm{Da}$ derselbe aber die anderen Angaben völlig ignorirt ${ }^{2}$ ) und dabei auch nicht den Schatten eines Beweises für seine Behauptungen beibringt, so dürfte es wohl kaum erforderlich sein, seine Feststellungen im einzelnen

1) Diescs Salz war zusammengespart und hatte Monate lang über Schwefelsäure gestanden, wobei es vielleicht $2 \mathrm{Mol}$. Krystallwasser verloren hat; denn die drei anderen isomeren Barytsalze enthalten 3 Mol. Krystallwasser.

2) Ein einziges Citat findet sich. Dasselbe ist jedoch irrthümlich. Herx Wichelhaus sagt nämlich vom $\beta$-Methylnaphtalin: „Es schmilzt, wie Fittig und Liebmann bei der Synthese fanden, bei . . . “" Die erste und einzige Synthese des $\beta$-Methylnaphtalins rührt aber von Léon Roux (Ann. Chim. [6] 12, 289) her, während Fittig, wie schon erwähnt, der erste war, dem die Synthese des $\alpha$-Methylnaphtalins gelang. (Ann. Chem. 155, 114.) 
zu widerlegen. Herr Wichelhaus beweist ja nicht eimmal, dass er überhaupt Methylnaphtaline in Händen gehabt hat. Er giebt z. B. nicht eine einzige Analyse, weder von den Kohlenwasserstoffen, noch von irgend einem Derivat! Und trotzdem sagt er unter anderem von den Methylnaphtalinen ${ }^{1}$ ): "Dieselben haben in reinem Zustande folgende Eigenschaften..." und ferner $\left.{ }^{2}\right)$ : „Beim Abkühlen auf $0^{\circ}$ erstarrt dasselbe $(\beta$ Methylnaphtalin) und ist nach zweimaligem Umkrystallisiren aus Alkohol ganz rein. (!) Der Schmelzpunkt wurde dann (!) bei $38^{\circ}$ gefunden," mit welchen Worten die ganze Abhandlung schliesst.

Herr Wichelhaus ist offenbar allen Ernstes der Meinung, durch Ausfrieren in Verbindung mit Absaugen die vollständige Trennung der Isomeren bewerkstelligt zu haben, - was bisher vergebens versucht ist - ohne jedoch irgend welche Spur eines Beweises auch nur anzustreben.

Hier einige weitere Behauptungen des Hrn. Wichelhaus: Er sagt z. B.: "dass die Verfahren der Trennung (der Methylnaphtaline) durch Destilliren und Ausfrieren unzureichend seien, weil fremde Beimengungen dadurch nicht entfernt werden ..... „Die den Methylnaphtalinen“, fährt er fort, "hartnäckig anhaftende Beimengung ist aber nicht, wie ich anfangs glaubte, eine (!) stickstoff- oder schwefelhaltige Verbindung, sondern ein dritter Kohlenwasserstoff, nämlich Diphenyl.

Bei einer (!) Nitrirung des durch Destilliren oder Ausfrieren gereinigten $\alpha$-Methylnaphtalins blieb Diphenyl unverändert in solcher Menge zurück, dass es aus Alkohol in farblosen Blättern vom Schmelzp. $71^{\circ}$, die mit unzweideutigem Ergebniss analysirt wurden, erhalten werden konnte."

Obwohl also die "Menge" Diphenyl offenbar schon zur Siedepunktsbestimmung nicht mehr ausgereicht hat, obwohl ferner Reingruber ${ }^{3}$, als er die Methylnaphtaline im Steinkohlentheer auffand, ebenfalls einen Körper vom Schmelzp. $71^{0}$ als Diphenyl ansah, der sich jedoch schliesslich als zusammengesetzt erwies, obwohl auch weiterhin das Vorkommen des Diphenyls im Steinkohlentheer theils behauptet, theils geleugnet wird, glaubt Hr. Wichelhaus in Folge „einer Nitrirung" und

1) Ber. 24, 3920 .

2) Das. S. 3921.

3) Ann. Chem. 206, 380. 
der Analyse mit dem "unzweideutigen Ergebniss" diese streitige Angelegenheit einfach durch die Vorschrift erledigen zu können ${ }^{1}$ ): „Man muss also, wenn man aus Steinkohlentheer reines Methylnaphtalin herstellen will, von Diphenyl trennen."

Zum Schlusse noch einige Worte über das von Herrn Wichelhaus empfohlene Verfahren zur Abscheidung von Diphenyl, resp. Reindarstellung von Methylnaphtalin vermittelst Pikrinsäure: Zunächst vergisst Er. Wichelhaus, dass in jedem aus Steinkohlentheer stammenden Methylnaphtalin ${ }^{2}$ ) geringe Quantitäten von Naphtalin, den Aethylnaphtalinen, den Dimethylnaphtalinen, den Aethylennaphtalinen stets unzweifelhaft enthalten sind. Diese und andere Beimengungen werden theilweise beim Ausfrieren abgeschieden, theilweise, besonders aus dem festen $\beta$-Methylnaphtalin, durch Umkrystallisiren entfernt. Das von Hrn. Wichelbaus zur Reindarstellung des $\beta$-Methylnaphtalins angegebene, sehr umständliche Verfahren vermittelst der Pikrate hat nicht den geringsten $Z_{w e c k}$ da alle die mit den Methylnaphtalinen verwandten, z. B. die vorher aufgeführten Kohlenwasserstoffe gleichfalls Pikrate bilden und gemäss der Vorschrift des Hrn. Wichelhaus auch nach Anwendung seiner Methode das $\beta$-Methylnaphtalin noch ,zweimal" umzukrystallisiren ist.

Diese seine Methode stammt übrigens von Hrn. Dr. R. Schneider, der dieselbe im technologischen Institute des Hrn. Wichelhaus sowohl zur Entfernung der Beimengungen als auch zur Trennung der beiden Isomeren angewendet hat, jedoch ebenso wie Hr. Wichelhaus in beiden Fällen mit negativem Ergebniss. Uebrigens stammt auch der in seiner Abhandlung beschriebene Apparat zum Ausfrieren nicht von Hrn. Wichelhaus.

1) Ber. 24, 3919.

2) Selbstverständlich kann nur das Rohmethylnaphtalin vom Siedepunkt $239^{\circ}-243^{\circ}$ ernsthaft in Betracht kommen, da ein gleichfalls von Hrn. Wichelhaus aufgeführtes Material vom Siedep. $233^{\circ}-262^{\circ}$ überhaupt nicht als Methylnaphtalin angesehen werden kann und, falls es nicht gerade centnerweise zur Verfügung steht (Hr. Wichelhaus hat ca. 2 Kilo gehabt), die Verarbeitung auf Methylnaphtalin durch fractionirte Destillation etc. gar nicht lohnt. 